\title{
Irreversible Exchange of Heat and Mass across Narrow Boundaries Under Specified Conditions
}

\author{
Jurgen M. Honig ${ }^{1} \&$ Elizabeth M. Duselis ${ }^{1}$ \\ ${ }^{1}$ Department of Chemistry, Purdue University, USA \\ Correspondence: Jurgen M. Honig, Department of Chemistry, Purdue University, 560 Oval Drive, West \\ Lafayette, IN 47907-2084, USA. Tel: 1-765-494-5279. E-mail: jmh@purdue.edu
}

Received: February 21, 2012 Accepted: July 13, 2012 Online Published: July 13, 2012

doi:10.5539/ijc.v4n4p7 URL: http://dx.doi.org/10.5539/10.5539/ijc.v4n4p7

\begin{abstract}
We extend here a set of earlier articles that deal with irreversible processes across a thin boundary separating a system from its surroundings. We consider the transfer of heat and material when the system and reservoir are held at constant volume, and the temperatures $(T)$ and chemical potentials $(\mu)$ differ by arbitrary amounts in the two parts of the compound system. Three distinct time variations for changing temperature and composition in the system are adopted. Although for any specified change of state the entropies associated with the transfer of heat and matter are quite different in the three cases, the overall entropy change remains the same, as is consistent with entropy being a function of state. The relation of the present approach to standard methodology is discussed.
\end{abstract}

Keywords: irreversible thermodynamics, transfer of heat and material across a thin boundary, entropy changes, time-dependent transfer mechanisms

\section{Background Information}

The extension of thermostatic to irreversible processes, nowadays termed extended thermodynamics, has been the subject of intensive research over roughly one century. A recurrent problem in the theoretical treatment has been the specification of temperature, as well as pressure, and entropy, under nonequilibrium conditions - a subject area that has been accorded a review on its own (Jou, Casas-Vázquez, \& Lebon, 1999). We merely draw attention to several discussions of the subject (Kaufmann, Muschik, \& Schirrmeister, 1996; Lebon, Jou, \& Casas-Vázquez, 1996; Jou, Casas-Vázquez, \& Lebon, 2001) which indicate the complexities involved in such a task. Among other matters, there is no unique formulation for the specification of temperature under nonequilibrium conditions which depends on the particular irreversible phenomenon under investigation. Nevertheless, a study of several special cases (Evans 1989; Jou \& Casas-Vázquez, 1992; Fort, 1997; Jou, CasasVázquez, \& Lebon, 2010) indicates that the difference between the equilibration temperature and its dynamical analog, at least as it pertains to the flow of heat, tends to be small, generally much less than $10 \%$.

In these circumstances it seemed appropriate to ignore the difference between static and dynamic temperatures and to concentrate on the investigation of the transfer of heat, work, and matter across a boundary separating a system from its surroundings under conditions where the two portions are at different temperatures, volumes, and chemical potentials. The situation is depicted in Figure 1 by a temperature $(T)$ profile that remains constant in the two parts of the system, but changes abruptly across the thin intervening boundary; a similar profile obtains for the pressure $(P)$, and chemical potential $(\mu)$.

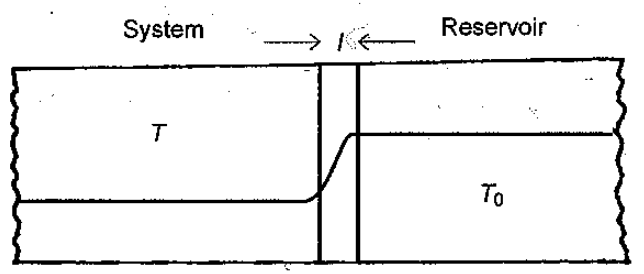

Figure 1. Sketch of a temperature profile for the combined system and reservoir at different temperatures $\mathrm{T}$ and $\mathrm{T}_{0}$. The temperature develops over only a small region 1 at the interface 
The standard analysis of the problem involves setting up linear phenomenological equations that relate fluxes and generalized forces, and imposing steady state conditions to identify the phenomenological coefficients. Here we proceed along different lines, based on a didactic article (Honig, 2011), that generalizes the standard thermodynamic functions of state, by including the contribution to the entropy arising from the irreversible transfers of heat, work, and material under the conditions represented in Figure 1. The processes occurring across the boundaries are characterized by specifying how the relevant control variables vary with time $(t)$, and then integrating to find the total entropy change over the entire time interval. The principal drawback of this procedure is the assumption that changes in $T, P$, and $\mu$ occur uniformly within the system except very close to the boundary. By convention, all processes within the reservoir will take place reversibly, and in such a manner that its intensive variables remain constant. For such a situation to be approximated experimentally one could work with a system that is either very tiny, and/or by assuming that all processes within the system are subject to a very fast relaxation rate. Alternatively, one may conceive of many reservoirs surrounding the system and acting in concert.

The subsequent analysis hinges on the differential of the path-dependent entropy change $d \theta$ that tracks the difference in entropy $S$ when the same element of a given process in the system is carried out irreversibly as opposed to reversibly. The corresponding entropy changes of the reservoir are designated by $d_{b} S_{0}$ and by $d_{a} S_{0}$ respectively; thus,

$$
d_{b} S_{0}-d_{a} S_{0}=d \theta
$$

Here we may set $d_{a} S_{0}=-d S(T, V, n)$, so that Eq. (1) reads

$$
d S(T, V, n)+d S_{0}\left(T_{0}, V_{0}, n_{0}\right)=d \theta .
$$

We now set up the differential equations for energy for the system and surroundings, as applicable to reversible processes; variables for the surroundings carry the subscript 0 .

$$
d E(S, V, n)=T d S-P d V+\mu d n ; \quad d E_{0}\left(S_{0}, V_{0}, n_{0}\right)=T_{0} d S_{0}-P_{0} d V_{0}+\mu_{0} d n_{0} .
$$

However, since $E$ and $E_{0}$ are functions of state we may reconstitute the above so as to apply to irreversible processes, by substituting for $d S_{0}$ from Eq. (2). We next invoke energy conservation for the compound system by setting $d E+d E_{0}=0$, impose volume conservation by setting $d V+d V_{0}=0$, and conservation of material by setting $d n+d n_{0}=0$. Lastly, we introduce the functional dependence of the entropy of the system by setting $d S=(\partial S / \partial T) d T+(\partial S / \partial V) d V+(\partial S / \partial n) d n$. This leads to the fundamental relation derived earlier by using a different approach (Honig, 2011) :

$$
T_{0} d \theta=\left(T_{0}-T\right)\left[\left(C_{V} / T\right) d T+(\partial P / \partial T) d V+\widehat{S} d n\right]-\left(P_{0}-P\right) d V+\left(\mu_{0}-\mu\right) d n,
$$

where $C_{V}$ is the heat capacity at constant volume, the appropriate Maxwell relation has been invoked, $\widehat{S}_{i} \equiv(\partial S / \partial n)_{T, V}$ is the differential entropy, and all other symbols retain their conventional significance.

In what follows we now assume special operating conditions in which the individual volumes are held fixed, leaving

$$
d \theta=\left(1-T / T_{0}\right)\left[\left(C_{V} / T\right) d T\right]+\left[\left(1-T / T_{0}\right) \hat{S}+\left(\mu_{0} / T_{0}\right)\left(1-\mu / \mu_{0}\right)\right] d n .
$$

With $T, V$, and $n$ as control variables, the relevant function of state is the Helmholtz free energy, for which $\hat{A} \equiv \mu=\hat{E}-T \hat{S}$. Eq. (5) may then be rewritten in the form

$$
d \theta=d \theta_{T}+d \theta_{n}=\left(1-T / T_{0}\right)\left[\left(C_{V} / T\right) d T\right]+\left[\hat{S}+\mu_{0} / T_{0}-\hat{E} / T_{0}\right] d n,
$$

where $\hat{E} \equiv(\partial E / \partial n)_{T, V}$ is the differential energy.

For definiteness we adopt the Berthetelot gas as a working substance in both parts of the compound unit; the equation of state for the system is specified by

$$
P(T, V, n)=n R T / V+n^{2} R T b / V^{2}-n^{2} a / V^{2} T,
$$

where $a$ and $b$ are materials-specific parameters. The energy derivative with respect to volume is then found from the caloric equation of state as

$$
(\partial E / \partial V)=T(\partial P / \partial T)-P=2 n^{2} a / V^{2} T,
$$


which may be integrated to yield

$$
\begin{gathered}
E(T, V, n)=E\left(T_{i}, V, n_{i}\right)+3 n R T / 2-2 n^{2} a / V T ; \quad C_{V}=3 n R / 2+2 n^{2} a / V T^{2} \\
\hat{E}=\hat{E}\left(T_{i}, V, n_{i}\right)+3 R T / 2-4 n a / V T .
\end{gathered}
$$

The entropy of the system (at constant volume) at temperature $T$ relative to the initial temperature $T_{i}$ is specified via

$$
S(T, V, n)=\int\left(C_{V} / T\right) d T=(3 n R / 2) \ln \left(T / T_{i}\right)-\left(n^{2} a / V\right)\left(1 / T^{2}-1 / T_{i}^{2}\right)+S\left(T_{i}, V, n_{i}\right),
$$

where $E\left(T_{i}, V, n_{i}\right), S\left(T_{i}, V, n_{i}\right)$ are the energy and entropy at initial temperature $T_{i}$ and mole number $n_{i}$, while the energy and entropy are to be evaluated at a temperature $T$ and mole number $n$. Then

$$
\left.\hat{S}(T, V, n)=(3 R / 2) \ln \left(T / T_{i}\right)-(2 n a / V)\left(1 / T^{2}-1 / T_{i}^{2}\right)+\hat{S}\left(T_{i}, V, n_{i}\right)\right) .
$$

With Eqs. (6) and (9) the entropy contribution associated with the temperature variation is given by

$$
\theta_{T}=\int(3 n R / 2 T) d T-\int\left(3 n R / 2 T_{0}\right) d T+\int\left(2 n^{2} a / V T^{3}\right) d T-\int\left(2 n^{2} a / V T^{2} T_{0}\right) d T .
$$

The contribution associated with the transfer of matter is specified via the second bracketed term in Eq. (6) as

$$
\Theta_{n}=\int\left[\frac{3 R}{2} \ln \frac{T}{T_{i}}-\frac{3 R T}{2 T_{0}}-\frac{2 n a}{V}\left(\frac{1}{T^{2}}-\frac{1}{T_{i}{ }^{2}}-\frac{2}{T T_{0}}\right)+\frac{\mu_{0}}{T_{0}}-\frac{\hat{E}}{T_{0}}\left(T_{i}, V, n_{i}\right)+\hat{S}\left(T_{i}, V, n_{i}\right)\right] d n .
$$

This completes the specification of the fundamentals. In what follows we shall temporarily ignore the contributions arising from $\mu_{0} / T_{0}-\hat{E}\left(T_{i}, V, n_{i}\right) / T_{0}+\hat{S}\left(T_{i}, V, n_{i}\right)$, which remain unchanged in the operations outlined in the next section.

\section{Specification of Entropy}

To determine the contributions of irreversible processes to the entropy of the combined unit we selected three different paths, by introducing time $t(0 \leq t \leq \tau)$ as a parameter and adopting three types of time dependence. We assume that $T_{i} \leq T \leq T_{f} \leq T_{0} ; n_{i} \leq n \leq n_{f} \leq n_{0}$.

\subsection{Case 1}

We set $T=T_{i} \exp \left(k_{T} t\right), \quad T_{f} / T_{i}=\exp \left(k_{T} \tau\right), \quad n=n_{i} \exp \left(k_{n} t\right), \quad n_{f} / n_{i}=\exp \left(k_{n} \tau\right), k_{T} / k_{n}=\ln \left(T_{f} / T_{i}\right) / \ln \left(n_{f} / n_{i}\right)$.

Insertion of these relations into Eq. (12) and carrying out the required integrations is a tedious but straightforward process and leads to the following contribution to the entropy arising from the transfer of heat associated with the temperature change:

$$
\theta_{1 T}=\theta_{1 T a}+\theta_{1 T b}+\theta_{1 T c}+\theta_{1 T d},
$$

where the first two terms on the right apply to the ideal gas case (via the first two terms in Eq. (12)), and the second set involves the additional corrections arising from the Berthelot equation of state. We find that

$$
\begin{gathered}
\theta_{1 T a} \equiv \frac{3 R}{2} \frac{k_{T}}{k_{n}}\left(n_{f}-n_{i}\right) ; \quad \theta_{1 T b} \equiv-\frac{3 R}{2 T_{o}} \frac{k_{T} / k_{n}}{1+k_{T} / k_{n}}\left(n_{f} T_{f}-n_{i} T_{i}\right) ; \\
\theta_{1 T c} \equiv \frac{a}{V} \frac{k_{T} / k_{n}}{1-k_{T} / k_{n}}\left[\frac{n_{f}^{2}}{T_{f}^{2}}-\frac{n_{i}^{2}}{T_{i}^{2}}\right] ; \quad \theta_{1 T d} \equiv-\frac{2 a}{V T_{0}} \frac{k_{T} / k_{n}}{2-k_{T} / k_{n}}\left[\frac{n_{f}^{2}}{T_{f}}-\frac{n_{i}^{2}}{T_{i}}\right] .
\end{gathered}
$$

Similarly, on applying this case to Eq. (13), corresponding to the transfer of matter, we obtain

$$
\theta_{1 n}=\theta_{1 n a}+\theta_{1 n b}+\theta_{1 n c}+\theta_{1 n d}
$$

where the first two terms relate to the contribution involving terms in $1 / T_{0}$ (excepting $\hat{E} / T_{0}$ ), as specified by 


$$
\theta_{1 n a}=-\frac{3 R}{2 T_{0}} \frac{1}{1+k_{T} / k_{n}}\left(T_{f} n_{f}-T_{i} n_{i}\right) ; \quad \theta_{1 n b}=\frac{4 a}{V T_{0}} \frac{1}{2-k_{T} / k_{n}}\left(\frac{n_{f}^{2}}{T_{f}}-\frac{n_{i}^{2}}{T_{i}}\right),
$$

and the remaining terms read

$$
\theta_{1 n c}=\frac{3 R}{2} \frac{k_{T}}{k_{n}}\left(n_{f} \ln \frac{n_{f}}{n_{i}}+\left(n_{i}-n_{f}\right)\right) ; \theta_{1 n d}=-\frac{a}{V}\left[\left(\frac{k_{T} / k_{n}}{1-k_{t} / k_{n}}\right)\left(\frac{n_{f}^{2}}{T_{f}^{2}}-\frac{n_{i}^{2}}{T_{i}^{2}}\right)+\frac{n_{f}^{2}}{T_{i}^{2}}-\frac{n_{i}^{2}}{T_{i}^{2}}\right] .
$$

\subsection{Case 2}

We set $T=T_{i} \exp \left(k_{T} t\right), \quad T_{f} / T_{i}=\exp \left(k_{T} \tau\right), \quad n=n_{i}\left(1+k_{n} t\right), \quad n_{f} / n_{i}=1+k_{n} \tau, \quad k_{T} / k_{n}=\ln \left(T_{f} / T_{i}\right) /\left(n_{f} / n_{i}-1\right)$.

Proceeding as before, for the contribution to the entropy from the temperature difference we write

$$
\theta_{2 T}=\theta_{2 T a}+\theta_{2 T b}+\theta_{2 T c}+\theta_{2 T d},
$$

where the first two terms involve the ideal gas contribution, and the others, the Berthelot portion. We obtain from Eq. (12)

$$
\begin{aligned}
& \theta_{2 T a} \equiv \frac{3 R}{4} \ln \frac{T_{f}}{T_{i}}\left[n_{f}+n_{i}\right] ; \quad \theta_{2 T b}=-\frac{3 R T_{i}}{2 T_{0}} n_{i}\left\{\left(1-\frac{k_{n}}{k_{T}}\right)\left(\frac{T_{f}}{T_{i}}-1\right)+\frac{k_{n}}{k_{T}}\left(\frac{T_{f}}{T_{i}} \ln \frac{T_{f}}{T_{i}}\right)\right\} ; \\
& \theta_{2 T_{c}} \equiv \frac{a}{V} n_{i}^{2}\left\{\begin{array}{l}
-\left(\frac{1}{T_{f}^{2}}-\frac{1}{T_{i}^{2}}\right)+\left(\frac{k_{n}}{k_{T}}\right)\left[\frac{1}{T_{f}^{2}} \ln \frac{T_{i}^{2}}{T_{f}^{2}}-\left(\frac{1}{T_{f}^{2}}-\frac{1}{T_{i}^{2}}\right)\right] \\
{\left[\left(\frac{n_{f}}{n_{i}}-1\right)^{2} \frac{1}{T_{f}^{2}}-\frac{1}{2}\left(\frac{k_{n}}{k_{T}}\right)^{2}\left[\frac{1}{T_{f}^{2}} \ln \frac{T_{i}^{2}}{T_{f}^{2}}-\left(\frac{1}{T_{f}^{2}}-\frac{1}{T_{i}^{2}}\right)\right]\right]}
\end{array}\right\} . \\
& \theta_{2 T d} \equiv \frac{2 a}{V T_{0}} n_{i}^{2}\left\{\begin{array}{l}
-\left(\frac{1}{T_{f}}-\frac{1}{T_{i}}\right)+2\left(\frac{k_{n}}{k_{T}}\right)\left[\frac{1}{T_{f}} \ln \frac{T_{i}}{T_{f}}-\left(\frac{1}{T_{f}}-\frac{1}{T_{i}}\right)\right] \\
+\left(\frac{n_{f}}{n_{i}}-1\right)^{2} \ln \frac{T_{i}^{2}}{T_{f}^{2}}-2\left(\frac{k_{n}}{k_{T}}\right)^{2}\left[\frac{1}{T_{f}} \ln \frac{T_{i}}{T_{f}}-\left(\frac{1}{T_{f}}-\frac{1}{T_{i}}\right)\right]
\end{array}\right\} .
\end{aligned}
$$

As regards the transfer of matter as specified by Eq. (13), we find that

$$
\theta_{2 n}=\theta_{2 n a}+\theta_{2 n b}+\theta_{2 n c}+\theta_{2 n d},
$$

where the first two terms relate to the contribution involving terms in $1 / T_{0}$ (excepting $\hat{E} / T_{0}$ ), as specified by

$$
\theta_{2 n a}=-\frac{3 R}{2 T_{0}} n_{i} \frac{k_{n}}{k_{T}}\left(T_{f}-T_{i}\right) ; \quad \theta_{2 n b}=\frac{4 a n_{i}^{2}}{V}\left[-\frac{k_{n}}{T_{0} k_{T}}\left(\frac{1}{T_{f}}-\frac{1}{T_{i}}\right)+\frac{1}{T_{i}^{2}}\left(\frac{k_{n}}{k_{T}}\right)^{2}\left(\frac{T_{f}}{T_{i}} \ln \frac{T_{f}}{T_{i}}-\frac{T_{f}}{T_{i}}+1\right)\right]
$$

and the remaining terms read

$$
\begin{gathered}
\theta_{2 n c}=\frac{3 R}{4}\left(\ln \frac{T_{f}}{T_{i}}\right)\left(n_{f}-n_{i}\right) \\
\theta_{2 n d}=\frac{a n_{i}^{2}}{V}\left\{\left[\frac{k_{n}}{k_{T}}\left(\frac{1}{T_{f}^{2}}-\frac{1}{T_{i}^{2}}\right)\right]-\left[\frac{1}{2}\left(\frac{k_{n}}{k_{T}}\right)^{2}\left(\frac{1}{T_{f}^{2}} \ln \frac{T_{i}^{2}}{T_{f}^{2}}-\frac{1}{T_{f}^{2}}+\frac{1}{T_{i}^{2}}\right)\right]+\frac{1}{n_{i}^{2}}\left(\frac{n_{f}^{2}}{T_{i}^{2}}-\frac{n_{i}^{2}}{T_{i}^{2}}\right)\right\} .
\end{gathered}
$$

\subsection{Case 3}

Set $T=T_{i}\left(1+k_{T} t\right), \quad T_{f} / T_{i}=\left(1+k_{T} \tau\right), n=n_{i}\left(1+k_{n} t\right), \quad n_{f} / n_{i}=1+k_{n} \tau, \quad k_{T} / k_{n}=\left(T_{f} / T_{i}-1\right) /\left(n_{f} / n_{i}-1\right)$. 
For this case the integrals associated with the Berthelot corrections become extremely unwieldy. We therefore opted to examine only the contributions associated with the ideal gas. Proceeding as before we find that

$$
\begin{gathered}
\dot{\theta}_{3 T}^{g}=\frac{-3 R}{4 T_{0}}\left(n_{f}-n_{i}\right)\left(T_{f}-T_{i}\right) . \\
\theta_{3 n}^{i g}=\frac{3 R}{2}\left[n_{i} \frac{k_{T}}{k_{n}} \ln \frac{T_{f}}{T_{i}}+\left(n_{f}-n_{i}\right) \ln \frac{T_{f}}{T_{i}}-\left(n_{f}-n_{i}\right)\right] .
\end{gathered}
$$

\section{Discussion}

We call attention to an important point that was also encountered in earlier treatments of other irreversible phenomena (Hoehn \& Honig, 2011; Honig \& Hoehn, 2011): Even though the $\theta_{T}$ and $\theta_{n}$ contributions to the total entropy are vastly different for the three cases treated above, the sum of these two terms is invariant, which is in consonance with the requirement that entropy be a function of state. We find that in all cases the entropy production for an ideal gas is given by

$$
\theta^{i g}=\frac{3 R}{2} n_{f} \ln \frac{T_{f}}{T_{i}}-\frac{3 R}{2 T_{0}}\left(n_{f} T_{f}-n_{i} T_{i}\right)
$$

If we include the Berthelot corrections we obtain

$$
\theta=\frac{3 R}{2} n_{f} \ln \frac{T_{f}}{T_{i}}-\frac{3 R}{2 T_{0}}\left(n_{f} T_{f}-n_{i} T_{i}\right)+\frac{a}{V} n_{f}^{2}\left(\frac{1}{T_{i}^{2}}-\frac{1}{T_{f}^{2}}\right)+\frac{2 a}{V T_{0}}\left(\frac{n_{f}^{2}}{T_{f}}-\frac{n_{i}^{2}}{T_{i}}\right) .
$$

One must, however, not forget to take account of the contributions that have been neglected, namely

$$
\theta_{n}^{0}=\left[\mu_{0} / T_{0}-\frac{\hat{E}}{T_{0}}\left(T_{i}, V, n_{i}\right)+\hat{S}\left(T_{i}, V, n_{i}\right)\right]\left(n_{f}-n_{i}\right) .
$$

On the right hand side we determined the chemical potential via $\mu_{0}=\hat{E}_{0}-T_{0} \hat{S}_{0}$, where $\hat{E}_{0}, T_{0} \hat{S}_{0}$ were specified by replacing $T, V, n$ in Eqs. (9) and (11) with $T_{0}, V_{0}, n_{0}$ respectively; the terms in the initial configuration $T_{i}, V$, $n_{i}$ cancel against identical terms contained in $\mu_{0} / T_{0}$, because the material in the system and the reservoir is the same. Since the latter remains at constant temperature $T_{0}$ (and the volume remains fixed as well) only the change in mole number enters in the derivation. We obtain

$$
\theta_{n}^{0}=\left\{\left[\frac{3}{2} R-\frac{3}{2} R \ln \frac{T_{0}}{T_{i}}\right]-a\left(c_{0 f}+c_{0 i}\right)\left[\frac{1}{T_{0}^{2}}+\frac{1}{T_{i}^{2}}\right]\right\}\left(n_{f}-n_{i}\right),
$$

where $c_{0} \equiv n_{0} / V_{0}$ is the concentration of gas in the reservoir.

This completes the specification of the entropy change under the assumed condition for the exchange of heat and transfer of matter.

\section{Relation to Standard Methodology}

The present approach has its antecedent in early work (Bejan, 1997; Honig, 2008; Jarzynski, 1997; Kestin, 1996; Tolman \& Fine, 1948), who dealt with special aspects, as reviewed by Honig (2011). Earlier work along the present line of approach may be found in Ben Amotz and Honig (2006a; 2006b), Honig and Ben Amotz (2005; 2008), Hoehn and Honig (2011) and Honig and Hoehn (2011).

It is also of interest to relate the above methodology to standard procedures (de Groot \& Mazur, 1962; Gyarmati, 1970; Haase, 1990; Kondepudi \& Prigogine, 1998; Meixner, 1954; Mueller \& Ruggeri, 1993; Prigogine, 1967) for treating irreversible processes in discontinuous systems such as depicted in Figure 1. In this latter approach these authors focused attention on identifying the prevailing forces and fluxes for particular situations and then 
invoking a linear response of the various forces or fluxes to their the conjugate variables. This engenders the standard linear phenomenological equations subject to the Onsager reciprocity conditions. These relations are then solved under a variety of assumed steady state conditions, so as to analyze thermomolecular and mechanocaloric effects. In the present procedure emphasis is placed on investigating irreversible processes in discontinuous system whose path is specified by a predetermined time variation, which precludes the establishment of steady state conditions. It is nevertheless possible to link the current approach to standard methodology. Toward that end we reformulate Eq. (4) under constant volume conditions as

$$
T_{0} d \theta=(-\Delta T) \cdot\left(\frac{\partial S}{\partial T}\right)_{V, n} d T-\Delta T \cdot\left(\frac{\partial S}{\partial n}\right)_{T, V} d n-\Delta \mu \cdot d n .
$$

Now take derivatives with respect to time for an infinitesimal step, to write

$$
T_{0} d \theta=-\Delta T \cdot\left(\frac{\partial S}{\partial T}\right)_{V, n} \frac{d T}{d t}-\Delta T \cdot\left(\frac{\partial S}{\partial n}\right)_{T, V} \frac{d n}{d t}-\Delta \mu \cdot \frac{d n}{d t}=-(\Delta T)\left[\left(\frac{\partial S}{\partial t}\right)_{V, n}+\left(\frac{\partial S}{\partial t}\right)_{T, V}\right]-\Delta \mu \cdot \frac{d n}{d t} .
$$

Now introduce the entropy density, $\vartheta$, where for a junction of cross section $A$ and length $l$

$$
\dot{d} \dot{\vartheta}=\left(1 / T_{0}\right)\left[-\frac{\Delta T}{l} \cdot \frac{1}{A}\left\{\left(\frac{\partial S}{\partial t}\right)_{V, n}+\left(\frac{\partial S}{\partial t}\right)_{T, V}\right\}-\frac{\Delta \mu}{l} \cdot \frac{1}{A} \frac{d n}{d t}\right] .
$$

In the limit of a very thin junction, and on adopting the standard formulation for entropy flux $J_{S}$ and particle flux, $J_{n}$, we obtain ( $\nabla$ is the gradient operator)

$$
d \dot{\vartheta}=\frac{1}{T_{0}}\left[-\nabla T \cdot J_{S}-\nabla \mu \cdot J_{n}\right] .
$$

This expression is the standard formulation for the entropy density flux in terms of the conjugate force-flux pairs. Thus, the present formulation is seen to be a variant of the ordinary treatment of irreversible thermodynamics procedure, and is therefore subject to the extremum and variational principles and Hamilton's principle, as discussed by Gyarmati (1970).

\section{References}

Bejan, A. (1997). Advanced Engineering Thermodynamics (2nd Ed.). New York: Wiley, p. 135.

Ben Amotz, D., \& Honig, J. M. (2006a). The Rectified Second Law of Thermodynamics. J. Phys. Chem. B, 110, pp. 19966-19972. http://dx.doi.org/10.1021/jp0621631

Ben Amotz, D., \& Honig, J. M. (2006b). Average Entropy Dissipation in Irreversible Mesoscopic Processes. Phys. Rev. Lett., 96, pp. 020602/1-4. http://dx.doi.org/10.1103/PhysRevLett.96.020602

de Groot, S. R., \& Mazur, P. (1962). Non-Equilibrium Thermodynamics. North Holland, Amsterdam

Evans, D. J. (1989). On the Entropy of Nonequilibrium States. J. Stat. Phys., 57, pp. 745-758. http://dx.doi.org/10.1007/BF01022830

Fort J. (1997). Information-theoretical Approach to Radiative Transfer. Physica A, 243, pp. 275-303. http://dx.doi.org/10-1016/S0378-4371(97)00277-x

Gyarmati, I. (1970). Non-equilibrium Thermodynamics, Field Theory and Variational Principles. New York: Springer Verlag.

Haase, R. (1990). Thermodynamics of Irreversible Processes. New York: Dover.

Hoehn, R., \& Honig, J. M. (2011). Analysis of Irreversible Processes across Narrow Junctions. Acta Phys. Polon. A, 119, pp. 323-327.

Honig, J. M. (2008). Thermodynamics, Principles Characterizing Chemical and Physical Processes, 3rd Ed., Amsterdam: Academic Press, Chap. 6.

Honig, J. M. (2011). Thermodynamics of Irreversible Processes Across a Boundary; Elementary Principles. Appl. Phys. Res., 3, 3-11. http://dx.doi.org/10.5539/apr.v3n2p3 
Honig, J. M., \& Ben Amotz, D. (2005). The Analysis of Spontaneous Processes Using Equilibrium Thermodynamics. J. Chem. Ed. 83, pp 132-137. http://dx.doi.org/10.1021/ed083p132

Honig, J. M., \& Ben Amotz, D. (2008). Rectification of Thermodynamic Inequalities as a Means of Characterizing Irreversible Phenomena. Chem. Educator, 13, pp. 220-226. http://dx.doi.org/10.1333/s00897082138a

Honig, J. M., \& Hoehn, R. (2011). Entropy of Irreversible Processes across a Boundary. Open J. Chem. Eng., 5, pp. 1-6. http://dx.doi.org/10.2174/1874123101105010001

Jarzynski, C. (1997). Non-Equilibrium Equality for Free Energy Differences. Phys. Rev. Lett., 78, pp. 2690-2693. http://dx.doi.org/10.1103/PhysRevLett.78.2690

Jou, D., \& Casas-Vázquez, J. (1992). Possible Experiments to Check the Reality of a Nonequilibrium Temperature. Phys. Rev. A, 45, 8371-8373. http://dx.doi.org/10.1103/PhysRevA.45.8371

Jou, D., Casas-Vázquez, J., \& Lebon, G. (2010). Extended Irreversible Thermodynamics (4th Ed.). Berlin: Springer Verlag, Chap. 3. http://dx.doi.org/10.11007/978-90-481-3074-0

Jou D., Casas-Vázquez, J., \& Lebon, G. (1999). Extended Thermodynamics Revisited (1988-1998). Rep. Progr. Phys., 62, pp. 1052-1056. http://dx.doi.org/10.1088/0034-4885/62/7/201

Kaufmann, M., Muschik W., \& Schirrmeister D. (1996). Entropy and Entropy Generation. Shiner J. S., Ed. Kluwer Academic, Dordrecht, Chap. 1.

Kestin, J. (1966). A Course in Thermodynamics. Blaisdell, Waltham, MA, Chap. 13

Kondepudi, D., \& Prigogine, I. (1998). Modern Thermodynamics, from Heat Engines to Dissipative Structures, Wiley, Chichester. Chaps. 15-17.

Lebon, G., Jou, D., \& Casas-Vázquez, J., (1996). Entropy and Entropy Generation. Shiner J. S., Ed. Kluwer Academic, Dordrecht, pp. 37-45

Meixner, J. (1954). Thermodynamik der Irreversiblen Prozesse. Meizner, Aachen.

Mueller, I., \& Ruggeri, T. (1993). Extended Thermodynamics. New York: Springer Verlag. http://dx.doi.org/10.1007/978-1-4684-0447-0

Prigogine, I. (1967). Introduction to Thermodynamics of Irreversible Processes. New York: Wiley.

Tolman, R. C. \& Fine, P. C. (1948). On the Irreversible Production of Entropy. Rev. Mod. Phys., 20, pp. 51-77. http://dx.doi.org/10.1103/RevModPhys.20.51 